\title{
FORUM
}

Anne Menzel

\section{Zwischen Herrschaftswissen und Irrelevanz? Feldforschung und das Ringen mit der Policy-Relevanz}

Feldforschung in Krisengebieten und Nachkriegsgesellschaften ist aus der aktuellen Friedens- und Konfliktforschung, die zunehmend Policy-relevant sein soll und will, nicht mehr wegzudenken. Feldforschung ist von zentraler Bedeutung für die Erarbeitung von Wissen für und über Peacebuilding-Maßnahmen; und angesichts oft enttäuschender Peacebuilding-Ergebnisse, die einen stetigen Bedarf an Verbesserungswissen produzieren, nimmt die Nachfrage nach Daten aus den jeweiligen Interventionskontexten stetig zu. Mein Beitrag wirft die Fragen auf, inwieweit und inwiefern Feldforschung unter dem Imperativ der Policy-Relevanz auch abseits der Produktion von Verbesserungswissen kritische Potenziale bereithält und wie diese sich realisieren lassen. Als eine vielversprechende Möglichkeit, das Ringen mit der Policy-Relevanz produktiv zu gestalten, wird die feldforschungsbasierte Suche nach Perspektivwechseln und die Konfrontation von Peacebuilding-»Wahrheiten « vorgeschlagen und anhand von zwei Forschungsbeispielen illustriert.

Schlagworte: Kritik, Peacebuilding, produktive Irritation, Herrschaftswissen, Feldforschung

\section{Einleitung}

Feldforschung in Krisengebieten und Nachkriegsgesellschaften gehört mittlerweile zur gängigen Praxis und Normalität der deutschen Friedens- und Konfliktforschung. Mehr noch: Feldforschung scheint sogar einen ihrer ganz besonders anziehenden und faszinierenden Aspekte auszumachen. Nach Gastvorträgen, die ich in den letzten Jahren im Rahmen von Lehrveranstaltungen zur Friedens- und Konfliktforschung gehalten habe, habe ich beispielsweise oft erlebt, dass mein jeweils »eigentliches « Vortragsthema in den anschließenden Diskussionen in den Hintergrund rückte; stattdessen wurde ich detailliert zu Feldforschungserfahrungen befragt, auf die ich im Vortrag nur am Rande eingegangen war. Die Nachfrage nach Feldforschungsverfahrenswissen - etwa zur Kontaktaufnahme mit Interview- und Ge- 
sprächspartner_innen, zu Interviewtechniken und zu den Möglichkeiten und Grenzen teilnehmender Beobachtung - ist groß. Der Hintergrund ist oft, dass Studentinnen und Studenten selbst mit dem Gedanken spielen, eine Feldforschung durchzuführen, sei es im Rahmen eines Promotionsvorhabens oder auch schon für die Masterarbeit. Ich weiß aus zahlreichen Gesprächen und vor allem auch aus eigener Erfahrung, dass in solchen Überlegungen und Plänen ganz unterschiedliche Motivationen eine Rolle spielen und zusammenkommen können: Da ist zum einen ein gewisses $\mathrm{Ma} ß$ an Fernweh und Abenteuerlust, das zwar selten offen thematisiert wird (vermutlich aus Sorge, es könnte sich frivol und dem Ernst der Lage nicht angemessen anhören), ohne welches solche Pläne aber wohl gar nicht in Erwägung gezogen würden; dann ist da der Wunsch, das jeweilige Forschungsthema gründlich zu erschließen und tatsächlich zu verstehen, nicht nur an der Oberfläche zu verharren, sondern in Kontakt zu den Menschen zu treten, um deren Realitäten es in der Friedens- und Konfliktforschung geht; hinzu kommt noch das Bestreben, über die eigene Forschungsarbeit zumindest einen kleinen »Beitrag « zu leisten, dessen konkrete Konturen nicht selten vage bleiben, der aber oft im weitesten Sinne etwas mit Friedenspraxis- und Politikberatung zu tun haben soll; und nicht zuletzt ist die Bereitschaft zu Feldforschung - mit allen persönlichen Risiken und sozialen Kosten, die ein längerer, oft immens bereichernder und zugleich potenziell verstörender und zuweilen nachhaltig verändernder Forschungsaufenthalt mit sich bringen kann auch eine Karriereentscheidung. Viele aktuelle Themen und Fragestellungen der Friedens- und Konfliktforschung sind ohne die Bereitschaft zu Feldforschung in Krisengebieten und Nachkriegsgesellschaften kaum bearbeitbar; noch dazu müssten gerade begehrte Projektstellen, auf denen oft vor allem Doktorandinnen und Doktoranden die benötigten Felddaten erheben sollen, ohne die Bereitschaft zu Feldforschung gleich vom Bewerbungszettel gestrichen werden.

Dass Feldforschung während des Studiums und in der Promotionsphase zunehmend üblich ist und sowohl Studierende als auch ihre Betreuerinnen und Betreuer vor erhebliche Herausforderungen stellt, ist in dieser Zeitschrift bereits in zwei Forumsbeiträgen, von Susanne Buckley-Zistel (2012) und Felix Koltermann (2013), diskutiert worden. Mein Beitrag soll eine Ergänzung zu ihren Überlegungen darstellen. Während Buckley-Zistel und Koltermann in erster Linie Anforderungen an und notwendige Kompetenzen von Studierenden und Betreuenden thematisieren, will ich hier zum einen auf die Hintergründe des Feldforschungs-Booms in der Friedens- und Konfliktforschung und zum anderen auf kritische Potenziale eingehen, die Feldforschung in und für die Friedens- und Konfliktforschung bereithält. Ich verlagere damit den Fokus von den Herausforderungen der Planung, Umsetzung und Betreuung von Feldforschung hin zu den Fragen, weshalb Feldforschung in 
Krisengebieten und Nachkriegsgesellschaften eigentlich so normal geworden ist (in dem Sinne, dass sie als Methode nicht länger erstaunlich ist oder grundsätzlich besonderer Begründung bedarf), wie sich in dieser Normalität kritische Perspektiven erschließen lassen und was genau dann das Kritische an ihnen sein könnte. Mein Beitrag knüpft dabei an einen Vortrag an, den ich im März 2014 im Rahmen der dritten Konferenz junger Wissenschaftler_innen der Arbeitsgemeinschaft für Friedens- und Konfliktforschung (AFK) gehalten habe, die mit der Frage bzw. dem Ausruf aufmachte: »What the FuK?! Kritische Perspektiven in der/auf die Friedensund Konfliktforschung «. ${ }^{1}$ Auf der Konferenz wurde deutlich, dass die Suche nach und das Ringen um kritische Perspektiven gerade auch junge Wissenschaftlerinnen und Wissenschaftler umtreibt, die (wie auch ich) akademisch in einem Umfeld »aufgewachsen« sind, das zunehmend Policy-relevant sein soll und will. PolicyRelevanz als Anforderung und Anspruch strahlt eine moralisch unterlegte Notwendigkeit aus, der nur schwer zu entkommen ist - und die zugleich Anlass zu nagenden Zweifeln gibt. Die Überlegungen, die ich im Folgenden ausführe, sind nicht zuletzt vor dem Hintergrund meines eigenen Ringens mit der Policy-Relevanz formuliert, das sich im Kontext von Forschungsprojekten zu und Feldforschungserfahrungen in Bosnien und Herzegowina und Sierra Leone - während des Studiums, in der Promotionsphase und danach als freiberufliche Beraterin für eine PeacebuildingNichtregierungsorganisation (NGO) - abgespielt hat und weiter abspielt.

\section{Peacebuilding, Verbesserungswissen und der Feldforschungs-Boom}

Der Feldforschungs-Boom in der Friedens- und Konfliktforschung ist ein noch relativ neues Phänomen. Ich selbst habe es zwar nicht mehr aktiv miterlebt, aber ich habe zumindest noch Echos einer Zeit vernehmen können, in der Feldforschung noch keine Normalität war. Ich erinnere mich etwa an Kommentare, in denen ältere Teilnehmer sich im Jahr 2007 im Rahmen einer Veranstaltung des AFK-Arbeitskreises »Theorie« und auf der später im selben Jahr veranstalteten AFK-Nachwuchstagung darüber austauschten, dass »die Jungen« jetzt ja wohl alle solche Feldforschung machen würden; da käme man ja auch gar nicht mehr drum herum. Es ist gerade diese Entwicklung, die Überlegungen und Vorschläge wie die, die von Buckley-Zistel (2012) und Koltermann (2013) in ihren Forumsbeiträgen angestellt und diskutiert worden sind, so dringend nötig macht. Denn es kann wohl kein Zweifel daran bestehen, dass Studierende und junge Wissenschaftler_innen sich idea-

1 Die Konferenz fand am 2. und 3. April 2014 in der Evangelischen Akademie Villigst statt. Für weitere Informationen siehe: http://www.afk-web.de/arbeitskreis-nachwuchs/3-konferenz-jungerwissenschaftler-innen-der-afk-2014.html (letzter Zugriff: 30.6.2014). 
lerweise nicht ohne fundierte methodische Vorkenntnisse, ohne Bewusstsein über oft kaum vermeidbare ethische Konflikte und Dilemmata oder gar ohne eine Abwägung persönlicher Risiken und angemessene Betreuung und Absicherung auf Feldforschung einlassen sollten. Dass es in diesen Hinsichten noch einiges an Verbesserungspotenzialen gibt und zugleich stets Abwägungen zwischen, einerseits, Eigenverantwortlichkeit und selbständiger Durchführung geplanter Forschungsvorhaben und, andererseits, enger Betreuung und Sicherheitsregularien nötig sind, machen die Beiträge von Buckley-Zistel und Koltermann deutlich. Mir geht es hier jedoch noch um eine andere Problematik und Herausforderung.

Es ist wohl keine allzu gewagte These, dass der Feldforschungs-Boom zumindest zu erheblichen Anteilen damit zusammenhängt, dass die Friedens- und Konfliktforschung zunehmend Wissen für und über Maßnahmen liefern soll und will, über die friedensbildend - so die Annahme beziehungsweise das oft unhinterfragt gelassene Framing - in Kriegs- und Nachkriegskontexte eingegriffen wird. Friedensbildende Eingriffe (oder auch Peacebuilding, ich verwende die Begriffe hier synonym) ${ }^{2}$ verlangen zum einen nach detaillierten Hintergrundinformationen über lokale Konfliktdynamiken und Konfliktparteien, die idealerweise aus intensiver und direkter Beschäftigung mit Strukturen, Wahrnehmungen und Einstellungen vor Ort gewonnen werden; und zum anderen produzieren sie einen permanenten Bedarf an Verbesserungswissen, das sich idealerweise unmittelbar in Policy-Empfehlungen überführen lassen soll. Da die Ergebnisse friedensbildender Eingriffe häufig hinter den in sie gesteckten Erwartungen zurückbleiben und zudem mit jeder neuen Verbesserungsempfehlung neue Probleme auftreten oder antizipiert werden können, sind die Themen und Fragen für die Erarbeitung von Verbesserungswissen schier unendlich. Hier nur einige Beispiele: Wie lassen sich Prozesse des Umgangs mit der gewaltsamen Vergangenheit so gestalten, dass bestimmte Gewalterfahrungen und »Wahrheiten« über den Konflikt nicht systematisch ausgeklammert werden, Opfer keine Re-Traumatisierung durchmachen müssen und Täterinnen und Täter keine Racheakte zu fürchten haben? Wie müssen Entwaffnungs- und Reintegrationsprozesse vor dem Hintergrund bisheriger Erfahrungen angelegt werden, um Remobilisierungen und kriminelle Nachkriegsgewalt nachhaltig zu verhindern und auch den besonderen Bedürfnissen von Frauen und Mädchen in bewaffneten Gruppen gerecht zu werden? Wie lassen sich

2 Unter friedensbildenden Maßnahmen, Eingriffen bzw. Interventionen und Peacebuilding verstehe ich hier-sehr breit angelegt - alle militärischen und politischen Programme, Maßnahmen und Reformen, die von externen Akteuren in Kriegs- und Nachkriegskontexten umgesetzt werden (meist, in je unterschiedlichen Ausmaßen, unter Einbeziehung verschiedener lokaler Akteure, etwa Regierungen, Armeen, Ministerien, NGOs etc.) und damit begründet und gerechtfertigt werden, dass sie Frieden ermöglichen und/oder fördern sollen. 
gegebenenfalls geeignete nichtstaatliche Partner für die Bereitstellung von Sicherheitsleistungen identifizieren, wenn staatliche Akteure nicht kooperieren oder verlässlich versagen - und kann dies tatsächlich eine gangbare Option darstellen? Und so weiter und so fort. Um solches Verbesserungswissen zu erarbeiten, ist Feldforschung bei den »intervenierten Menschen « (Daxner 2010: 75) oft unerlässlich. Ihre unbedingte Notwendigkeit ergibt sich der Verbesserungslogik nach, die ultimativ ein in intendierter Weise wirksames und erfolgreiches Peacebuilding in Aussicht stellt, zudem aus einer speziellen Verantwortung der Forschenden. Diese Verantwortung wird von Hannah Neumann konsequent auf den Punkt gebracht als »responsibility to understand « bezeichnet:

»The responsibility to understand, much like other concepts such as $>$ sustainability or `gender sensitivity«, should serve as a main concept to guide research as well as inform policy formulation and the conceptualisation of programmes in the course of future peace- and statebuilding. Peacebuilding practitioners and researchers have a responsibility to understand the complexity of societies in which they intervene or which they research about. Social dynamics are always complex and post-war dynamics are even more complex « (Neumann 2013:30).

Verantwortungsbewussten Forscherinnen und Forschern bleibt demnach keine andere Option, als die Produktion von Verbesserungswissen voranzutreiben. Denn, so argumentiert beispielsweise der bekannte Peacebuilding-Forscher Roland Paris, eine vertretbare Alternative zur wissensbasierten Verbesserung von Peacebuilding gebe es nicht: »[S]uch missions, in spite of their many flaws, have done more good than harm « (Paris 2010: 338; Anm. AM) - eine Aussage, die sich in ihrer Pauschalität mit sozialwissenschaftlichen Methoden weder aussagekräftig belegen noch falsifizieren lässt. Nichtsdestotrotz schlussfolgert Paris, jede Abkehr vom »existierenden Peacebuilding-Projekt « sei grob fahrlässig und habe absehbar schreckliches Leid zur Folge, »because the failure of the existing peacebuilding project would be tantamount to abandoning tens of millions of people to lawlessness, predation, disease and fear « (Paris 2010: 338).

Wenn es aber doch gelingt, sich von dem moralischen Druck der Verbesserungslogik ein Stück weit freizumachen (etwa angeregt durch abweichende und herausfordernde Erfahrungen oder Lektüre), drängt sich als nächstes die Frage auf, wie unter Bedingungen drängender Policy-Relevanz und angesichts von Peacebuilding-Verbesserungsnotwendigkeiten überhaupt sinnvoll anders geforscht werden kann. Ist die Produktion von Verbesserungswissen, das stets zumindest ein gewisses $\mathrm{Ma}$ an - notwendigerweise konstruktiver - Kritik an der aktuellen PeacebuildingPraxis enthält, womöglich doch alternativlos? Oder lassen sich auch abseits der 
Produktion von Verbesserungswissen kritische Perspektiven auftun und kritische Potenziale freisetzen?

\section{Zwischen Herrschaftswissen und Irrelevanz?}

Die Frage nach kritischen Perspektiven abseits der Produktion von Verbesserungswissen stellt sich vor allem angesichts nagender Zweifel daran, dass die internationale Peacebuilding-Praxis tatsächlich Frieden fördert und überhaupt zur Friedensförderung geeignet ist. Solche Zweifel sind in den letzten Jahren oft unter dem Schlagwort einer Kritik am »liberal peace « formuliert worden - einer politikprägenden Weltanschauung, der zufolge es keine Alternative zu Interventionen in Kriegs- und Nachkriegskontexte gibt, über die diese mehr oder weniger grob nach dem Vorbild westlicher (und als friedlich verstandener) Staaten und Gesellschaften um-modelliert werden sollen. Entsprechende Maßnahmen und Politiken, so wird kritisiert, sind oft selbst autoritär, gewaltgestützt, werden lokalen Friedensanforderungen und -hindernissen nicht gerecht, schaffen Abhängigkeiten von westlichen Geberstaaten, internationalen Organisationen und der NGO-Industrie, übergehen lokale gesellschaftliche Ressourcen oder zerstören sie sogar und bleiben insgesamt weit hinter dem erklärten Ziel einer umfassenden und dauerhaften Friedensbildung zurück, zu deren Realisierung sie demnach gar nicht geeignet sind (vgl. etwa Richmond 2009; Kühn 2012; Mac Ginty/ Richmond 2013). Dass dennoch am »liberal peacebuilding « festgehalten wird, so diagnostizieren einige, habe vor allem mit den Sicherheitsinteressen westlicher Staaten zu tun. Diese würden danach streben, in Krisengebieten - nach quasi-kolonialherrschaftlicher Manier - für sie einigermaßen kontrollierbare und durchschaubare Verhältnisse (oder Verhältnisse, die für kontrollierbar und durchschaubar gehalten werden) herzustellen; dies nicht zuletzt vor den Hintergründen der Terrorismusbekämpfung und der Einhegung von Flüchtlingsströmen, Armutsmigration und transnationaler Kriminalität (vgl. etwa Duffield 2001; 2007; 2010; Kühn 2012).

Andere Autorinnen und Autoren, die zu Unterscheidungszwecken die »Arbeitsprozessorientierten « genannt werden können, zeigen auf, dass konfliktive politische Interessen und widersprüchliche organisationale Logiken dazu führen, dass erarbeitetes Verbesserungswissen von Peacebuilding-Akteuren bislang gar nicht systematisch zur Maßnahmenverbesserung genutzt wird (vgl. etwa Lipson 2007; Campbell 2008; Benner/Rotmann 2008). Zu dieser Problematik kommt aus arbeitsprozessorientierter Perspektive noch hinzu, dass die Übertragbarkeit von Verbesserungswissen - von seinem jeweiligen Erarbeitungskontext auf einen anderen und damit auch sein praktischer Nutzen (das tatsächliche Verbesserungspotenzial) zwangsläufig ungewiss bleiben. Dass in einem jeweiligen Kontext erarbeitete 
lessons learned oder best practices in einem anderen Kontext Verbesserungen ermöglichen können, mag im Vorhinein angenommen werden. Mit Sicherheit abschätzbar ist es jedoch nie. Mit Blick auf Reformen innerhalb des Systems der Vereinten Nationen (UN), die an dem Ideal einer wissensbasierten und zugleich organisational optimierten, nämlich koordinierten und integrierten Steuerung orientiert sind, wendet Susan Campbell deshalb skeptisch ein, dass stattdessen eher eine explorative Herangehensweise sinnvoll wäre:

»In reality, a country's actual war-to-peace trajectory is only revealed as the country advances through each stage of its transition. For post-conflict efforts to truly support war-to-peace transitions, they would have to be prepared to >discover the stages of each transition as they appeared, possibly as a result of the intervention's influence but heavily dependent on the social and institutional realities of the country and the politics of its new leadership « (Campbell 2008: 563).

Allerdings erscheint eine derart explorative Vorgehensweise in den heute existierenden organisationalen Zusammenhängen völlig illusorisch, da diese in erster Linie »angebotsorientiert « angelegt sind. Dies bedeutet, dass internationale Organisationen, staatliche Agenturen und Ministerien und internationale NGOs in erster Linie Maßnahmen implementieren, zu denen sie bereits anerkannte Expertise entwickelt haben und die einigermaßen fertig und einsatzbereit sind. Es ist eben nicht der Fall, dass Maßnahmen immer wieder neu und an speziellen »Bedürfnissen« der jeweiligen Interventionskontexte orientiert ausgearbeitet werden, was eine explorative Neuausrichtung organisationsstrukturell zumindest noch eher zulassen würde (vgl. Campbell 2008: 561; Campbell/Kaspersen 2008: 476).

Eine mögliche Antwort auf die Frage, warum dann eigentlich überhaupt Verbesserungswissen nachgefragt wird, formuliert David Chandler (2007). Er argumentiert, Verbesserungswissen fungiere mittlerweile als Ersatz für politische Visionen und werde permanent benötigt, um gewichtig erscheinende Policy-Statements abgeben zu können, die jedoch - abgesehen von Hinweisen auf die Komplexität der Peacebuilding-Aufgaben und auf die, leider, stets ungewissen Erfolgsaussichten, denen mit noch mehr Verbesserungswissen sowie mit Koordination und Integration begegnet werden müsse - wenig Substanz und politischen Willen und noch weniger tatsächliche Verbesserungsaussichten für die jeweils Intervenierten enthalten. Zudem warnt Chandler davor, Interventionen im Namen des Friedens (oder im Namen von Sicherheit und Entwicklung, die dauerhaften Frieden bringen sollen) pauschal mit einer quasi-kolonialen Durchsetzung westlicher Interessen gleichzusetzen. 
Denn diese Interpretation impliziere deutlich mehr Vision und politischen Willen aufseiten des Westens, als tatsächlich und effektiv erkennbar seien:

»With little connection between policy rhetoric and political commitment or accountability for policy outcomes, policy-making and judgments of policy success have been highly self-referential. This desire to prioritize rhetoric over policy responsibility is also reflected in the shift away from the focus on particular policy issues to broader and more declaratory projects, such as >saving Africa<, ’preventing state failure $<$, not just ending conflicts but >resolving the causes of conflicts $<$, or >eradicating poverty<. [...] While non-Western states are the focus of declarations by Western leaders and international institutions, the lack of connection between rhetoric and strategic policymaking indicates less a demand to revive colonial practices of domination and more the antiforeign policy desire to see the non-Western world in symbolic rather than practical policy-making terms« (Chandler 2007: 373).

Wenn diese verschiedenen Ausführungen und Einwände als begründet - oder zumindest als ausreichend beunruhigend - angesehen werden, ergibt sich für die $\mathrm{Po}$ licy-relevante Forschung zu und für Peacebuilding eine skurrile Situation: nämlich eine, in der sie irgendwo zwischen der Produktion von Herrschaftswissen und praktischer Irrelevanz (bei gleichzeitig immer wieder erklärter Policy-Relevanz) eingeklemmt ist. Denn zum einen kann dann zumindest nicht ausgeschlossen werden, dass im Namen des Friedens implementierte Interventionspolitiken doch letztlich auf Formen von Gefahreneinhegung hinauslaufen, die in erster Linie an westlichen Sicherheitsinteressen oder zumindest an einer Weltanschauung orientiert sind, in der nichts anderes als westlichen Verhältnissen ähnliche institutionelle und gesellschaftliche Strukturen als Stabilitätsgaranten infrage kommen. Zum anderen ist zumindest ungewiss, ob Verbesserungswissen tatsächlich im intendierten Sinne (also friedensrelevant) nützlich ist - oder von relevanten Akteuren (so sie die jeweiligen Forschungsarbeiten tatsächlich jemals zu sehen bekommen und zur Kenntnis nehmen) auch nur zu nutzen versucht wird. Verbesserungswissen wäre in dieser Situation so ziemlich jeder kritischen Kraft beraubt. ${ }^{3}$

3 Dieser Vorschlag einer aktuellen Situationsdefinition der Produktion von Verbesserungswissen für Peacebuilding stellt aus meiner Sicht eine Ergänzung zu der Situationsdefinition dar, die ich vor einigen Jahren gemeinsam mit Sven Chojnacki in einem Artikel zum Thema »Peacebuilding: am Scheideweg - oder in der Sackgasse« formuliert habe (vgl. Chojnacki/Menzel 2011). Darin haben wir, in etwa im Einklang mit einigen »Arbeitsprozessorientierten«, vor allem dargelegt, dass über die Wirkung von Peacebuilding zu wenig gewusst wird und Zeit und Raum übergreifend überhaupt zu wenig gewusst werden kann, als dass sich aus vermeintlichem Verbesserungswissen tatsächlich Steuerungsmöglichkeiten für die Wirkung von Peacebuilding ableiten lassen würden. 
Inwieweit diese Situationsbeschreibung für zutreffend zu halten ist, können Forscherinnen und Forscher letztlich nur für sich selbst einzuschätzen versuchen. Ich selbst habe über ein andauerndes Ringen mit der Policy-Relevanz hinaus noch keinen abschließenden Standpunkt gefunden; und ich vermute, dass ein solcher Standpunkt, falls ich ihn irgendwann einmal definieren sollte, auch wieder in Grauzonen angesiedelt wäre. Angesichts solcher Ambivalenz und Graustufigkeit kann am ehesten vielleicht eine von Michel Foucault formulierte Geisteshaltung weiterhelfen, der zufolge nicht alles böse, »sondern [...] alles gefährlich ist, was nicht dasselbe ist wie böse. Wenn alles gefährlich ist, dann haben wir immer etwas zu tun" (Foucault 1994: 268). »Zu tun« ist in diesem Fall, über den Tellerrand des Verbesserungswissens hinauszuschauen und nach alternativen kritischen Perspektiven und Potenzialen Ausschau zu halten, die - und genau darauf will ich hier in erster Linie hinaus - auch und gerade Feldforschung bereithalten kann.

\section{Produktive Irritation durch Perspektivwechsel}

Inwieweit und inwiefern Feldforschung kritische Potenziale bereithält - und ob sie überhaupt kritische Potenziale bereithält und worin diese bestehen könnten - wird in ihren »Heimatdisziplinen «, in der Ethnologie und Sozial- und Kulturanthropologie, spätestens seit den 1980er Jahren kontrovers diskutiert. ${ }^{4}$ Die Ausgangs- und Angriffspunkte dieser Debatten bestanden in den kolonialen oder zumindest kolonialgedanklichen Verstrickungen von Feldforschung, die lange in erster Linie zur Erschließung und Übersetzung »exotischer « (»unzivilisierter « und vermeintlich von westlichen Einflüssen noch »unverfälschter«) Kulturen eingesetzt worden war, und in der mit diesen Forschungszielen verknüpften epistemologischen Überzeugung, »exotische« Kulturen mithilfe von Feldforschung und der textlichen Darstellung ihrer Ergebnisse tatsächlich authentisch wiedergeben zu können. Die Forschungsansprüche hatten sich dabei in einem durchaus konfliktgeladenen Spagat bewegt: zwischen der Produktion von Herrschaftswissen, das zuweilen explizit von praktisch-kolonialherrschaftlichem Nutzen sein sollte (vgl. etwa Gijswijt-Hofstra 2014: 5-6), und Bewahrungswissen, das einen (oft herrschaftskritisch gedachten) Wert an sich darstellen sollte und von dem Anspruch motiviert war, »to study the natives >before they disappeared « (Scheper-Hughes 1993: 27). An dieser Stelle ist kein Platz, um die Debatten der letzten Jahrzehnte auch nur ansatzweise nachzuzeichnen.

4 Vgl. etwa Clifford/Marcus (1986); Marcus/Fischer (1986); Spiro (1996); MacClancy (2002); Hoffman (2004); Zenker/Kumoll (2010); Jensen (2014). Für Diskussionen des zunehmenden »Imports« von Feldforschung in andere sozialwissenschaftliche Disziplinen vgl. Vrasti (2008) und Wedeen (2010). 
Stattdessen muss es hier genügen, einige zentrale Konsequenzen dieser Debatten im Groben festzuhalten. Zu diesen gehört, erstens, eine zunehmend verbreitete Sensibilität dafür, dass Feldforschung aufgrund ihres andauernden Anspruchs, Fremdes zumindest verständlicher und Unbekanntes zumindest zugänglicher oder überhaupt erst sichtbar zu machen, stets und zwangsläufig mit dem Risiko einhergeht, Herrschaftswissen über die Erforschten zu liefern, welches sich durchaus zu ihrem Nachteil auswirken kann; ${ }^{5}$ zweitens die Überzeugung, dass eine tatsächlich ganz authentische Erfahrung und Wiedergabe der erforschten Kulturen (oder Praktiken, Beziehungen, Imaginationen etc.) kaum möglich ist, da Forscherinnen und Forscher das Feld stets gefiltert durch die eigenen Erfahrungshintergründe, Perspektiven und Erkenntnisinteressen wahrnehmen und ihre eigene vielschichtige soziale Situiertheit dabei nie vollständig abschütteln können - zumal ihnen auch in ihren jeweiligen Forschungskontexten oft viele der je feldspezifisch typischen Erfahrungen vorenthalten werden bzw. erspart bleiben (vgl. etwa Scheper-Hughes 1993: 28-29; Chapkis 2010: 487-489); und drittens eine disziplinäre Erschütterung und Verunsicherung, für die es bislang nicht »die eine « gute Lösung gibt, anhand derer sich Feldforschung dann eindeutig mit gutem Gewissen und der Gewissheit einer wahrhaft kritischen Perspektive praktizieren ließe - zumal auch die Frage, worin der Geltungsanspruch und die Zielrichtung einer solchen Kritik bestehen könnte, bislang nicht (und auch absehbar nicht) abschließend und allgemeingültig beantwortbar ist (vgl. etwa Jensen 2014).

Aber auch wenn es nicht»die eine «befriedigende und endgültige Lösung gibt, existieren durchaus Ideen und Vorschläge dazu, wie mit und über Feldforschung Kritik realisiert werden kann. Am vielversprechendsten erscheinen mir dabei Herangehensweisen, die auf eine Art von Kritik abzielen, die ohne überlegene und als herrschaftsfrei implizierte Standpunkte auskommt, ohne »views from nowhere « (Donna Haraway zitiert in Jensen 2014: 338), von denen ausgehend andere Standpunkte dann, im »kritischen Ergebnis«, kategorisch als »falsch« und verherrschaftlicht entlarvt

5 Eine Fundamentalkritik, die Sozial- und Kulturanthropologie und ihre zentralen Methoden - Feldforschung und die textliche Darstellung ihrer Ergebnisse - als direkte Fortsetzung globaler Machtund Ausbeutungsverhältnisse mit anderen Mitteln porträtiert, hat Edward Said (1989) formuliert. Er argumentiert, dass keinerlei methodische Sorgfalt die imperialen Verwendungszwecke des erarbeiteten Wissens über fremde Kulturen im Zaum halten kann: »The fetishization and relentless celebration of >difference< and >otherness< can therefore be seen as an ominous trend. It suggests [...] the heedless appropriation and translation of the world by a process that for all its protestations of relativism, its displays of epistemological care and technical expertise, cannot easily be distinguished from the process of empire " (Said 1989: 213-214). Dass diese Kritik weiterhin erstgenommen werden muss, wird beispielsweise daran deutlich, dass militärische Interventionsakteure an ethnographisch (über Feldforschung) erarbeitetem Wissen zur Optimierung von »Aufstandsbekämpfungsstrategien« (Counterinsurgency) interessiert sind (vgl. etwa Forte 2011; Plemmons/Albro 2012). 
würden (vgl. auch Latour 2004: 237-241). In einer ohne »views from nowhere« auskommenden Kritik geht es vielmehr darum, die Pluralität von Erfahrungen und Perspektiven aufzuzeigen und so die Situiertheit von Vorstellungen und Argumenten wie beispielsweise die des Arguments der Alternativlosigkeit von Peacebuilding (siehe oben, Paris 2010) - sichtbar zu machen. Ihr Ziel ist keine »abschließende Dekonstruktion «, in der schließlich alles relativ oder nichts anderes als die eigene kritische Perspektive mehr »echt « und berechtigt erscheinen würde, sondern die Konstruktion von empirisch begründbaren Alternativen zu bekannten »Wahrheiten« (zu Vorstellungen, die tendenziell als gegeben hingenommen und nicht hinterfragt werden) und zu vermeintlichen Alternativlosigkeiten. Solche Kritik setzt keine Endpunkte. Stattdessen verkompliziert sie vermeintliche Eindeutigkeit und ist auf dieser Grundlage gerade nicht als peinliche Zurechtweisung und Akt des Ausschlusses, sondern als Einladung zur Diskussion zu verstehen (vgl. etwa Scheper-Hughs 1993: 30; Latour 2004: 231-232, 247-248).

Das Medium, über welches solche Kritik mit Feldforschung und in der Interpretation feldforschungserhobener Daten realisiert werden kann, sind irritierende Perspektivwechsel, die auch dann noch erreichbar und textlich darstellbar sind, wenn die Erschließung lokaler Erfahrungen und Sichtweisen stets nur annäherungsweise möglich ist und das Feld nie wirklich ganz authentisch mit den Augen der »Eingeborenen « gesehen werden kann (going native). Tatsächlich ist es unter kritischen (oder um Kritikmöglichkeiten ringenden) Gesichtspunkten nicht tragisch, wenn Perspektivwechsel unvollkommen bleiben - solange sie immerhin eine ernsthafte Konfrontation der eigenen Kultur beziehungsweise der in der eigenen Kultur zumindest tendenziell »gegebenen « Standpunkte ermöglichen. ${ }^{6}$ Solche Perspektivwechsel-basierten Konfrontationen sind grundsätzlich über zwei miteinander verstrickte und stets produktiv kombinierbare Wege erreichbar: erstens indem Fremdes bekannt gemacht wird, wobei bestehende Vorstellungen über die Fremden konfrontiert und alternative, durch Feldforschung informierte Vorstellungen konstruiert werden; und zweitens indem Bekanntes verfremdet wird, wobei fremde Perspektiven genutzt werden, um das Bekannte zunächst überhaupt hinterfragen und dann neu konfigurieren zu können (vgl. etwa Marcus/Fischer 1986: 4, 137-141; Mac-

6 Letzteres wird am ehesten dann der Fall sein, wenn nichtsdestotrotz Bemühungen unternommen werden, an lokale Erfahrungen und Sichtweisen zumindest so nah wie möglich »heranzukommen«. Nancy Scheper-Hughes nennt diese Herangehensweise ») good enough〈 ethnography«: »We cannot rid ourselves of the cultural self we bring with us into the field any more than we can disown the eyes, ears, and skin through which we take in our intuitive perceptions about the new and strange world we have entered. Nonetheless [...] we struggle to do the best we can with the limited resources we have at hand - our ability to listen and observe carefully, emphatically, and compassionately« (Scheper-Hughes 1993: 28). 
Clancy 2002: 7-8; Stepputat 2012: 442-444). Um diese Wege und ihre kritischen Potenziale anschaulich zu machen, sind konkrete Anwendungsbeispiele hilfreicher als abstrakte Darlegungen. Bevor ich abschließend zu einem kurzen Fazit komme, werde ich im Folgenden deshalb zwei feldforschungsbasierte Studien schildern, die jeweils in unterschiedlicher Weise und an unterschiedlichen Forschungsgegenständen Perspektivwechsel und die Konfrontation mit Peacebuilding-»Wahrheiten « suchen.

Als ein Beispiel kann meine Dissertation »Was vom Krieg übrig bleibt: Unfriedliche Beziehungen in Sierra Leone « (Menzel 2015) dienen.7 Darin bin ich den Fragen nachgegangen, wer eigentlich aus welchen Gründen in Nachkriegs-Sierra Leone vor wem Angst hat, wer aus welchen Gründen gewaltbereit ist und ob die in Angst und Gewaltbereitschaft konstituierten unfriedlichen Beziehungen tatsächlich plausibel zwischen ehemaligen Kämpfern und der Zivilbevölkerung (denjenigen, die selbst nicht aktiv an Gewalttaten beteiligt waren) verortet werden können - so wie es für Sierra Leone und viele andere Nachkriegskontexte in der Peacebuilding-Praxis und -Forschung üblicherweise vorausgesetzt wird. ${ }^{8}$ Die Situierung »in der Peacebuilding-Praxis und -Forschung « stellte dabei gerade keine Abgrenzung dar, sondern schloss mein eigenes Denken von vornherein mit ein. Denn wohl nicht zuletzt aufgrund meiner Sozialisierung im Peacebuilding-Feld, auf das ich mich gegen Ende meines Studiums spezialisiert hatte, konnte auch ich mir zunächst (vor allem vor der Feldforschung) mit Blick auf Nachkriegs-Sierra Leone gar nichts anderes als unfriedliche Beziehungen zwischen Exkombattanten und der Zivilbevölkerung vorstellen. Anlass und Hintergrund der Forschungsfragen war jedoch eine in mir allmählich gewachsene Skepsis. Diese bezog sich auf die »Lösungsfixiertheit« der Peacebuilding-Praxis und -Forschung, die mir im Zuge einschlägiger Praktikums- und Feldforschungserfahrungen (in Bosnien und Herzegowina) und

7 Ich habe mich dazu durchringen müssen, hier als eines von zwei Beispielen ausgerechnet meine eigene Arbeit vorzustellen. Letztendlich halte ich dies aber für konsequent, da ich die Überlegungen, die ich in diesem Beitrag ausbreite, vor allem im Kontext der Arbeit an meiner Dissertation entwickelt habe. In jedem Fall aber hätte ich hier eigentlich gerne noch mehr Studien vorgestellt, aus Platzgründen musste ich mich jedoch auf zwei beschränken. Ich will hier dennoch zumindest noch eine Leseempfehlung für eine gerade erst ganz frisch erschienene Studie von Kathleen Jennings (2014) abgeben, in der sie Peacebuilding-»Wahrheiten « über das Verhältnis von Peacekeepern und Lokalen konfrontiert: »Service, Sex and Security: Gendered Peacekeeping Economies in Liberia and the Democratic Republic of the Congo«.

8 Ich schreibe hier deshalb nur von ehemaligen Kämpfern, weil ehemalige Kämpferinnen in dieser üblichen Voraussetzung zwar häufig mit benannt werden, zugleich ist die ihnen zugeschriebene Problematik aber eine, die nichtsdestotrotz eher auf der Seite der Zivilbevölkerung verortet wird. Mädchen und Frauen in bewaffneten Gruppen werden aus Peacebuilding-Perspektive eher als Opferdenn als Tätergruppe angesehen (vgl. ausführlich Menzel 2015: 51-54). 
intensiver Peacebuilding-Lektüre schon während des Studiums aufgefallen war. Kurzgefasst: Die Frage, zwischen wem und worin genau eigentlich friedensrelevante Probleme bestehen, wird für Nachkriegskontexte meist gar nicht gestellt. Stattdessen geht es um Lösungen (Peacebuilding-Maßnahmen), die stetig verbessert werden müssen. Mein Ausgangspunkt für die Untersuchung bestand dann in dem Verdacht, dass die in der Suche nach stetig verbesserten Lösungen längst als bekannt vorausgesetzten Problemdefinitionen - in meiner Untersuchung konkret die Exkombattanten/Zivilbevölkerung-Trennlinie - womöglich gar nicht »zutreffen « beziehungsweise einer Konfrontation mit lokalen Erfahrungen und Sichtweisen nicht standhalten würden.

Im Ergebnis legt mein Feldforschungsmaterial (bestehend aus Interviews, informellen Gesprächen und Beobachtungen, gesammelt von Januar bis Mai 2009 im Süden von Sierra Leone) in der Tat eine alternative Definition unfriedlicher Beziehungen nahe, die ohne eindeutige Trennlinien auskommen muss. In dieser alternativen Definition, die ich strikt auf Basis des gesammelten empirischen Materials formuliert (»empirisch definiert«) habe, bestehen unfriedliche Beziehungen zwischen einer Klasse von Wachsamen und einer Klasse »gefährlicher junger Männer «. ${ }^{9}$ Die Wachsamen, zu denen alle meine Interview- und Gesprächspartnerinnen und -partner ohne Ausnahme gezählt werden können, zeichnen sich durch insgesamt sehr ähnliche Wachsamkeitseinstellungen aus, die wiederum einer gemeinsamen »Sicherheitslage « Rechnung tragen, in der davon ausgegangen wird, dass in Sierra Leone letztlich jede/r Einzelne selbst für die eigene Sicherheit Sorge tragen muss. Trotz vielseitiger Unterschiede zwischen meinen Interview- und Gesprächspartnerinnen und -partnern (im Hinblick auf sozioökonomischen Status, Alter, Geschlecht und ethnische Zugehörigkeit) sind ihre Wachsamkeitseinstellungen (oder auch Bedrohungserwartungen) einhellig auf sozioökonomisch marginalisierte »gefährliche junge Männer« bezogen. Letztere sind weder notwendigerweise ehemalige Kämpfer noch notwendigerweise gewaltbereit; dafür sind auch sie zugleich Wachsame. Allerdings unterscheiden sie sich von »ungefährlichen« Wachsamen dadurch, dass sie gemäß einer lokal als plausibel empfundenen »Ästhetik der Gefährlichkeit« wie

9 Ich benutze den Klassenbegriff im Sinne der Sozialtheorie Pierre Bourdieus, also mit Blick auf gemeinsame bzw. ähnliche strukturelle Positionen in sozialen Feldern und gemeinsame bzw. ähnliche Merkmale und Einstellungen, die aus ähnlichen Erfahrungen resultieren - ohne dass diejenigen, die sich entlang von Positionen, Merkmalen und Einstellungen klassifizieren lassen, deshalb auch ein Gruppengefühl oder Klassenbewusstsein haben müssten (vgl. etwa Bourdieu 1987: 171-195). In der Klasse der »Gefährlichen«, von denen Gewalt erwartet wird, kommen Mädchen und Frauen auf Basis meines empirischen Materials nicht vor; es scheint, dass sie auch in Sierra Leone (vgl. Fußnote 8) aus generalisierenden Bedrohungserwartungen ausgenommen sind (vgl. ausführlich Menzel 2015: 282-288). 
gewaltbereite ehemalige Kämpfer aussehen - beziehungsweise auf Grundlage bestimmter Merkmale und Tätigkeiten in diese Kategorie einsortiert werden (vgl. auch Menzel 2011). Auf eine knappe Formel gebracht lässt sich meine empirische Definition unfriedlicher Beziehungen folgendermaßen zusammenfassen: Anders als es mit der Exkombattanten/Zivilbevölkerung-Trennlinie üblicherweise gedacht wird, bestehen unfriedliche Beziehungen zwischen Wachsamen und denjenigen, die für gefährlich gehalten werden - wobei letztere allerdings weder notwendigerweise ehemalige Kämpfer noch notwendigerweise gewaltbereit sind; und so sie tatsächlich gewaltbereit sind, resultieren ihre Gewaltbereitschaften zudem gerade nicht unmittelbar aus Kämpfervergangenheiten - falls sie überhaupt ehemalige Kämpfer sind.

Aus diesem Ergebnis lassen sich keine kontextübergreifenden Verallgemeinerungen ableiten, noch kann daraus der definitive Schluss gezogen werden, dass sich für andere Nachkriegsgesellschaften nicht womöglich doch eine klare Exkombattanten/Zivilbevölkerung-Trennlinie ziehen lässt. ${ }^{10}$ Aber es macht doch deutlich, dass schon allein die »Wissensbasis « (bestehend aus »bekannten« Problemdefinitionen), von der ausgehend lösungsfixiert als alternativlos deklariertes Verbesserungswissen erarbeitet wird, Schwachstellen aufweist. Die erarbeitete Kritik besteht in eben dieser Schwachstellen-Demonstration, in dem Aufzeigen einer empirisch begründeten alternativen Definition unfriedlicher Beziehungen und in der damit verbundenen Schlussfolgerung, dass Nachkriegskontexte auch anders als durch lösungsfixierte »Brillen« erkenntnisgewinnbringend untersucht werden können.

Das zweite Beispiel ist Finn Stepputats (2012) Studie »Knowledge Production in the Security-Development Nexus: An Ethnographic Reflection«, in der er die Produktion und Verwertung von Verbesserungswissen zum unmittelbaren Forschungsgegenstand macht. Das empirische Material für diese Untersuchung sammelte Stepputat in einem Feld, das nicht in erster Linie durch geographische Lokalität, sondern entlang des Produktionskontexts einer Policy-Analyse definiert wurde, an deren Erarbeitung er in seiner Eigenschaft als Senior Researcher am Danish Institute for International Studies (DIIS) selbst unmittelbar beteiligt war. Stepputat beschreibt dieses Feld folgendermaßen:

10 Allerdings argumentiere ich, dass ich dies für eher unwahrscheinlich halte, da die in zahlreichen Studien - und keinesfalls nur zu Sierra Leone - dokumentierten Komplexitäten und Dynamiken des Lebens und Überlebens unter Bedingungen kriegerischer Gewalt eher weniger dazu geeignet scheinen, eindeutige Trennlinien hervorzubringen, die dann das Leben in Nachkriegskontexten prägen. Dieses Argument begründe ich ausgehend von meiner Analyse des Entstehens unfriedlicher Beziehungen zwischen Wachsamen und »gefährlichen jungen Männern«, die ich im Anschluss an die empirische Definition dieser Beziehungen durchgeführt habe (vgl. ausführlich Menzel 2015: Kap. 7). 
»The >field ( or the >village $<$ of my study is not located among the >locals $<$ in the postcolony, which is often objectified as being at the root of problems of development and insecurity. Rather, my field is located in meeting rooms in Strandgade in Copenhagen during 2008-9, when policies for more coherent approaches to security governance were being developed in interconnected processes in a number of institutional hubs in the West « (Stepputat 2012: 441-442).

Das erklärte Ziel der Policy-Analyse, die von der dänischen Regierung in Auftrag gegeben worden war, sollte in der Erarbeitung von Vorschlägen für eine verbesserte interministerielle Koordination (zwischen Außen- und Verteidigungsministerium) und Integration ziviler und militärischer Programme und Vorgehensweisen im Kontext internationaler »peace-support operations « bestehen, $»)[\ldots]$ based on primarily the experience from the Danish engagement in Iraq and Afghanistan « (Stepputat 2012: 442). Die Hintergründe des Verbesserungsbedarfs, der über die Policy-Analyse bedient werden sollte, waren zum einen die öffentliche Unzufriedenheit mit der dänischen Präsenz im Irak und auch in Afghanistan, wo das zunächst als »humanitär« geframte Engagement bereits offensichtlich in Krieg umgeschlagen war; und zum anderen die in nationalen und internationalen Foren (etwa auch innerhalb von NATO und UN) vieldiskutierten Koordinationsanforderungen komplexer zivil-militärischer Operationen, deren erklärtes Ziel in der Eroberung der »hearts and minds « lokaler Bevölkerungen besteht. Die Policy-Analyse, die das DIIS in enger Abstimmung mit den relevanten Ministerien, der Armee und mit dem »K-committee« der dänischen Regierung vorzunehmen hatte, ${ }^{11}$ sollte vor diesen Hintergründen wissensbasierte praktische Anleitungen für die dänische Politik produzieren (vgl. Stepputat 2012: 442, 451).

Stepputat selbst beschreibt seine feldforschungsbasierte Studie als Reflexion auf Grundlage der Erfahrungen, die er - zunächst noch ganz ohne konkrete Forschungsabsicht - als teilnehmender Beobachter im Prozess der Erarbeitung der Policy-Analyse gesammelt hatte. Dass er sich im Arbeitsprozess überhaupt als teilnehmender Beobachter zu sehen begann (und zu sehen beginnen konnte), führt er auf eine produktive Entfremdung (»displacement«) zurück. Diese wiederum kam dadurch zustande, dass er aus seinem gewohnten akademischen Umfeld »displaced« wurde,

11 Das »K-committee« beschreibt Stepputat als »coordination committee consisting of the most influential ministers, including the prime minister and the ministers of foreign affairs, defence and finance« (Stepputat 2012: 442). Es scheint in Aufbau und Funktion in etwa mit dem National Security Council der US-Regierung vergleichbar zu sein. 
sodass er die eigentlich vertraute Wissensproduktionstätigkeit mit anderen Augen zu sehen begann:

»With my limited experience in doing relatively high-profile policy analysis, I felt somewhat like a stranger in a strange land. The encounter with the world of military institutions and practices was obviously new to me personally and resulted in a certain fascination with the new vocabulary and logics, but I was also struck by the differences between the worlds of researchers and policymakers« (Stepputat 2012: 445).

Die Kritik, die Stepputat mit seiner Studie offeriert, besteht in einer alternativen und am Beispiel der teilnehmend beobachteten Policy-Analyse empirisch begründeten Perspektive auf die Produktion und Verwertung von Verbesserungswissen. Anders als es die Verbesserungslogik - zumindest so wie sie in akademischen Kontexten üblicherweise verstanden wird - nahelegt, scheint die Produktion und Verwertung von Verbesserungswissen aus politikpraktischer Perspektive demnach nicht unbedingt und in erster Linie auf die Bewirkung von Verbesserungen vor Ort (bei den »intervenierten Menschen «, Daxner 2010: 75) ausgerichtet zu sein. Stepputat beschreibt, dass die Art und Weise, in der ministerielle Beamte, Armeeangehörige und Regierungsmitglieder das Projekt angingen, eher den Schluss nahelegt, dass mit solchen Verbesserungen ohnehin nicht gerechnet wurde; den Fragen »whether or not the projects on the ground resulted in reconstruction, development and security at the district level« (Stepputat 2012: 447) wurde kaum Aufmerksamkeit geschenkt. Insgesamt entstand für Stepputat sehr deutlich der Eindruck, dass es in der PolicyAnalyse so wenig wie möglich um Interventionseffekte gehen sollte. Er berichtet beispielsweise von Besprechungen, in denen die wissenschaftlichen Berater_innen dazu aufgefordert wurden, Überlegungen zur strategischen Antizipation möglicher »negativer Effekte« im Kontext zukünftiger Interventionen ganz aus dem Report zu streichen. Die Wissenschaftler_innen konnten schließlich einen Kompromiss aushandeln, der anscheinend bereits außergewöhnliche Zugeständnisse enthielt:

»In regard to the negative effects, we changed the wording to sunintended effects $\measuredangle$, even though the ministries were not happy with having us looking into the possible effects at all. Much later in the process, a civil servant who read the final terms of reference was struck by the fact that the word reffects $\ll$ had survived the negotiations« (Stepputat 2012: 447).

Alternativ zu den Implikationen der Verbesserungslogik schlägt Stepputat auf Basis seiner Erfahrungen vor, die Erarbeitung der Policy-Analyse als einen Prozess zu verstehen, der in erster Linie der internen Kommunikation, Abstimmung und 
Lösung von Verteilungskonflikten diente. Die beteiligten und von Koordinationsanforderungen unmittelbar betroffenen Akteure (ministerielle Beamte, Armeeangehörige und Regierungsmitglieder) rangen unter Verwendung wissenschaftlicher Expertise um Verständigung sowie um Standpunkte und Strategien, die einer zunehmend skeptischen Öffentlichkeit gegenüber politisch vertretbar sein würden und die die eigenen Kompetenzen, Ressourcen, Einflussmöglichkeiten etc. nicht gefährden würden. Stepputat selbst bringt seine alternative Interpretation folgendermaßen auf den Punkt:

»First and foremost, the analysis shows the degree to which knowledge production in this context is a negotiated and social process that may have more importance in bringing together a policy-interested community across fragmented policy fields than in actually guiding practice on the ground (Stepputat 2012: 440; Hervorh. AM).

Noch einmal kurz zusammengefasst haben beide Beispiele, sowohl meine Dissertation als auch Stepputats Studie, somit zweierlei gemeinsam: Zum einen entziehen sie sich dem moralischen Druck der Verbesserungslogik (was zumindest in meinem Fall ein ausgesprochen mühsamer und zweifelbeladener Prozess war) und zum anderen erarbeiten sie gerade kein Verbesserungswissen, sondern Alternativen zu Peacebuilding-»Wahrheiten «, die aus produktiver Irritation gewonnen werden und dazu geeignet sind, Leserinnen und Leser - und zwar gerade auch solche, die selbst Policy-relevant arbeiten - zur Teilhabe an produktiver Irritation einzuladen.

\section{Fazit}

Im Kontext des bislang zumindest zu erheblichen Anteilen von Policy-Relevanz angetriebenen Feldforschungs-Booms in der Friedens- und Konfliktforschung soll dieser Beitrag einem Ringen mit eben dieser Policy-Relevanz Ausdruck verleihen, die, im schlechtesten Fall, als vermeintlich alternativloses Leitbild einer Forschung fungiert, die zwischen Herrschaftswissen und Irrelevanz eingeklemmt bleibt. Dabei ging es mir in diesem Beitrag nicht (nur) darum, die Gelegenheit zu nutzen, meine eigenen Zweifel einmal systematisch auszubreiten; sondern vor allem auch darum, kritische Impulse, Bedürfnisse und Verstrickungen aufzugreifen, die auf der diesjährigen Konferenz junger Wissenschaftler_innen der AFK deutlich spürbar waren. Die Herausforderung besteht nun aus meiner Sicht darin, diese kritischen Impulse, Bedürfnisse und Verstrickungen produktiv weiterzutragen. Dies sollte unbedingt auf Arten und Weisen geschehen, die sich den durchaus immer noch üblichen (und auch auf der Konferenz noch beobachtbaren) Lagerbildungen zwischen »main- 
streamigen Policy-Relevanten« und »abgehobenen Kritiker_innen« entziehen. Das Feld ist in der Tat zu verstrickt, ambivalent und von zu vielschichtigen normativen Ansprüchen, Verantwortungen und Zweifelhaftigkeiten durchzogen, als dass es solchem Lagerdenken überlassen werden könnte. Eine abseits von Alternativlosigkeit gesuchte Erarbeitung von und Auseinandersetzung mit der Pluralität von Sichtweisen und Erfahrungen - bei den »Intervenierten « und in unseren eigenen Arbeitszusammenhängen - erscheint mir aussichtsreicher.

\section{Literatur}

Benner, Thorsten/Rotmann, Philipp 2008: Learning to Learn? UN Peacebuilding and the Challenges of Building a Learning Organization, in: Journal of Intervention and Statebuilding 2: 1, 43-62.

Bourdieu, Pierre 1987: Die feinen Unterschiede: Kritik der gesellschaftlichen Urteilskraft, Frankfurt a. M.

Buckley-Zistel, Susanne 2012: »Ich bin dann mal weg.« Feldforschung im Studium der Friedens- und Konfliktforschung, in: Zeitschrift für Friedens und Konfliktforschung 1: 2, 315-323.

Campbell, Susanna P. 2008: (Dis)integration, Incoherence and Complexity in UN Post-Conflict Interventions, in: International Peacekeeping 15: 4, 556-569.

Campbell, Susanna P./Kaspersen, Anja T. 2008: The UN's Reforms: Confronting Integration Barriers, in: International Peacekeeping 15: 4, 470-485.

Chandler, David 2007: The Security-Development Nexus and the Rise of >AntiForeign Policy<, in: Journal of International Relations and Development 10: 4, 362-386.

Chapkis, Wendy 2010: Productive Tensions. Ethnographic Engagement, Complexity, and Contradiction, in: Journal of Contemporary Ethnography 39: 5, 483-497.

Chojnacki, Sven/Menzel, Anne 2011: Peacebuilding: am Scheideweg - oder in der Sackgasse?, in: Politische Vierteljahresschrift 52: 3, 504-536.

Clifford, James/Marcus, George E. (Hrsg.) 1986: Writing Culture. The Poetics and Politics of Ethnography, Berkeley, CA.

Daxner, Michael 2010: Das Konzept von Interventionskultur als Bestandteil einer gesellschaftsorientierten theoretischen Praxis, in: Bonacker, Thorsten/Daxner, Michael/Free, Jan H./Zürcher, Christoph (Hrsg.): Interventionskultur. Zur Soziologie von Interventionsgesellschaften, Wiesbaden, 75-100.

Duffield, Mark 2001: Governing the Borderlands. Decoding the Power of Aid, in: Disasters 25: 4, 308-320. 
Duffield, Mark 2007: Development, Security and Unending War. Governing the World of Peoples, Malden, MA.

Duffield, Mark 2010: The Liberal Way of Development and the Development-Security Impasse. Exploring the Global Life-Chance Divide, in: Security Dialogue 41: 1, 53-76.

Forte, Maximilian C. 2011: The Human Terrain System and Anthropology. A Review of Ongoing Public Debates, in: American Anthropologist 113: 1, 149-151.

Foucault, Michel 1994: Zur Genealogie der Ethik. Ein Überblick über laufende Arbeiten (Interview mit Michel Foucault), in: Dreyfus, Hubert L./Rabinow, Paul: Michel Foucault. Jenseits von Strukturalismus und Hermeneutik, Weinheim, 265-292.

Gijswijt-Hofstra, Marijke 2014: Among the Mende in Sierra Leone. Letters from Sjoerd Hofstra (1934-36). Herausgegeben und übersetzt von Marijke GijswijtHofstra, Leiden.

Hoffman, Danny 2004: The Submerged Promise. Strategies for Ethnographic Writing in a Time of War, in: Anthropological Quarterly 77: 2, 323-330.

Jennings, Kathleen M. 2014: Service, Sex and Security. Gendered Peacekeeping Economies in Liberia and the Democratic Republic of the Congo, in: Security Dialogue 45: 3 (online vorab veröffentlicht im Juni 2014), 1-18.

Jensen, Casper B. 2014: Experiments in Good Faith and Hopefulness. Toward a Postcritical Social Science, in: Common Knowledge 20: 2, 337-362.

Koltermann, Felix 2013: Vom Hier und Dort - Lernen für die Praxis der Feldforschung. Eine Replik auf Susanne Buckley-Zistel, in: Zeitschrift für Friedensund Konfliktforschung 2: 1, 130-140.

Kühn, Florian P. 2012: The Peace Prefix. Ambiguities of the Word >Peaceく, in: International Peacekeeping 19: 4, 396-409.

Latour, Bruno 2004: Why Has Critique Run out of Steam? From Matters of Fact to Matters of Concern, in: Critical Inquiry 30: 2, 225-248.

Lipson, Michael 2007: Organized Hypocrisy?, in: European Journal of International Relations 13: 1, 5-34.

MacClancy, Jeremy 2002: Introduction. Taking People Seriously, in: MacClancy, Jeremy (Hrsg.): Exotic No More. Anthropology on the Front Lines, Chicago, IL, $1-14$.

Marcus, George E./Fischer, Michael M. J. 1986: Anthropology as Cultural Critique. An Experimental Moment in the Human Sciences, Chicago, IL.

Menzel, Anne 2011: Between Ex-Combatization and Opportunities for Peace. The Double-Edged Qualities of Motorcycle-Taxi Driving in Urban Postwar Sierra Leone, in: Africa Today 58: 2, 97-127. 
Menzel, Anne 2015: Was vom Krieg übrig bleibt. Unfriedliche Beziehungen in Sierra Leone, Bielefeld, i. E.

Neumann, Hannah 2013: Through the Eyes of the Locals. Two Post-war Communities and Their Struggles from War to Peace (Dissertation am Fachbereich Politik- und Sozialwissenschaften der Freien Universität Berlin), Berlin.

Paris, Roland 2010: Saving Liberal Peacebuilding, in: Review of International Studies 36: 2, 337-365.

Plemmons, Dena/Albro, Robert 2012: Practicing Ethics and Ethical Practice. The Case of Anthropologists and Military Humanitarians, in: Humanity: An International Journal of Human Rights, Humanitarianism, and Development 3: 2, 179-197.

Richmond, Oliver P. 2009: A Post-liberal Peace. Eirenism and the Everyday, in: Review of International Studies 35: 3, 557-580.

Richmond, Oliver P./Mac Ginty, Rogers 2013: The Local Turn in Peace Building. A Critical Agenda for Peace, in: Third World Quarterly 34: 5, 763-783.

Said, Edward W. 1989: Representing the Colonized. Anthropology's Interlocutors, in: Critical Inquiry 15: 2, 205-225.

Scheper-Hughes, Nancy 1993: Death without Weeping. The Violence of Everyday Life in Brazil, London.

Spiro, Melford E. 1996: Postmodernist Anthropology, Subjectivity, and Science. A Modernist Critique, in: Comparative Studies in Society and History, 38: 4, 759-780.

Stepputat, Finn 2012: Knowledge Production in the Security-Development Nexus. An Ethnographic Reflection, in: Security Dialogue 43: 5, 439-455.

Vrasti, Wanda 2008: The Strange Case of Ethnography and International Relations, in: Millenium - Journal of International Studies 37: 2, 279-301.

Wedeen, Lisa 2010: Reflections on Ethnographic Work in Political Science, in: Annual Review of Political Science 13: 1, 255-272.

Zenker, Olaf/Kumoll, Carsten 2010: Beyond Writing Culture. Current Intersections of Epistemologies and Representational Practices, New York, NY.

\section{Die Autorin}

Dr. Anne Menzel arbeitet als freiberufliche Beraterin, zuletzt für die NGO International Alert. Derzeit ist sie Gastwissenschaftlerin am Sonderforschungsbereich/Transregio $138 »$ Dynamiken der Sicherheit. Formen der Versicherheitlichung in historischer Perspektive«, Philipps-Universität Marburg und Justus-Liebig-Universität Gießen.

E-Mail: menzelanne@aol.com 